Erschienen in: Wimmer, Rainer (Hrsg.): Das 19. Jahrhundert: Sprachgeschichtliche Wurzeln des heutigen Deutsch. - Berlin, New York: de Gruyter, 1991. S. 185-201. (Institut für deutsche Sprache. JOACHIM SCHILDT

\title{
Zu einigen Entwicklungstendenzen im politischen Wortschatz der deutschen Arbeiterbewegung
}

\section{Zum Stand der Erforschung des politischen Wortschatzes der deutschen Arbeiterbewegung}

Wer sich die Geschichte der deutschen Sprache im 19. Jh. zum Untersuchungsgegenstand gewählt hat - jene Zeit also, in der die Voraussetzungen und Grundlagen für die gegenwärtige Sprachentwicklung gelegt wurden - kommt nicht umhin, sich auch mit der Frage zu beschäftigen, welchen Anteil die seit der industriellen Revolution stark anwachsende Arbeiterklasse - eingeschlossen die unterschiedlich organisierte Arbeiterbewegung mit ihren meist aus dem Bürgertum stammenden Theoretikern und Anführern - an den sich in diesem Jahrhundert vollziehenden sprachlichen Entwicklungsprozessen hatte.

Nun wurde die Sprachgeschichte des 19. Jh. ohnehin - mehrfach angemerkt - bisher von der Sprachgeschichtsforschung insgesamt stiefmütterlich behandelt, aber besonders groß ist das Defizit bei der Erforschung des Einflusses der Arbeiterklasse, speziell auch der Arbeiterbewegung, sowie Angehöriger unterprivilegierter Schichten auf die Sprachentwicklung. Ganz abgesehen davon, daß man heute überhaupt noch nicht imstande ist, präzisere Aussagen dazu machen, wie sich die schrittweise Aneignung des Standards durch breite Schichten des Volkes auf dessen Normengefüge auswirkte, fehlt es darüber hinaus an systematischen Untersuchungen auf allen Ebenen des Sprachsystems, so auch in der Lexik. $^{1}$ Der politische Wortschatz - um nur einige Bereiche herauszugreifen - der Gewerkschaftsbewegung ist genauso wenig beschrieben wie der von Parteiprogrammen oder politischen Schriften, die im Auftrage der in der 2. Jahrhunderthälfte gegründeten Arbeiterparteien verfaßt wurden. Die Sprache prominenter Theoretiker der Arbeiterbewegung ${ }^{2}$ -

1 Sehr verdienstvoll ist Brunner/Conze/Koselleck 1972-1984. Es stellt jedoch die Entwicklung zentraler Begriffe in den Mittelpunkt und geht auf sprachliche Fragen nur am Rande ein.

21952 wurde am damaligen "Institut für deutsche Sprache und Literatur" der Deutschen Akademie der Wissenschaften zu Berlin ein "Marx-EngelsWörterbuch" begründet. 1963 lagen eine vollständige Exzerption der Werke von Marx und Engels sowie Probeartikel vor. Die Sprachwissenschaftler des Akademieinstituts wollten ein Bedeutungswörterbuch des gesamten Wortschatzes, der sich in den Werken von Marx und Engels findet, erarbeiten. Das entsprach jedoch nicht den Vorstellungen der damaligen Führung der SED, die ein Sachwörterbuch zentraler Termini des Marxismus forderte. So 
ich nenne Bebel, Bernstein, Engels, Kautsky, Lassalle, Liebknecht, Luxemburg, Marx, Mehring - ist weitgehend unerforscht. Es sind bisher umfassendere Aussagen weder möglich zu der Frage, welchen Teil des politischen Wortschatzes der Arbeiterbewegung der Arbeiter ${ }^{3}$ kannte oder vielleicht auch aktiv verwendete, noch zu dem Problem, inwieweit dieser politische Wortschatz der Arbeiterbewegung schon Bestandteil der Allgemeinsprache war.

Aufgrund dieser Forschungslage muß ich mich daher darauf beschränken, einige typische Erscheinungen in der Entwicklung des politischen Wortschatzes der Arbeiterbewegung herauszugreifen. Ich demonstriere bei dieser Auswahl das sprachwissenschaftlich Relevante weitgehend an Beispielen aus Untersuchungen, die vor einigen Jahren im Zentralinstitut für Sprachwissenschaft der Berliner A kademie der Wissenschaften durchgeführt wurden. ${ }^{4}$ Bevor ich jedoch auf diese Fragen eingehe, halte ich es als Voraussetzung für das Verständnis für erforderlich, kurz auf einige Schwerpunkte in der Entwicklung der Arbeiterbewegung im 19. Jh. hinzuweisen.

Mit der Entstehung und Entwicklung des Industriekapitalismus in Deutschland in der ersten Hälfte des 19. Jh. begann sich auch die Arbeiterklasse, der 4. Stand, herauszubilden. In den dreißiger Jahren entstanden erste politische Vereinigungen, in denen sich Arbeiter organisierten. 1834 wurde in Paris die Geheimorganisation ${ }_{n}$ Bund der Geächteten" gegründet. Wandernde Handwerksgesellen, die zunächst noch die Mehrzahl der Mitglieder bildeten, verbreiteten in Deutschland die vom Bund herausgegebene Zeitschrift „Der Geächtete” und Flugschriften. 1838 wurde ein neuer proletarischer Geheimbund in Paris gegründet, der „Bund der Gerechten". Sein Programm war von W. Weitling verfaßt, einem Verteter des utopischen Sozialismus. 1846 wurde die Leitung des Bundes von Paris nach London, dem Schwerpunkt der Organisation, verlegt. Ihr traten $1847 \mathrm{~K}$. Marx und F. Engels bei. Der Bund beauftragte auf dem 1847 in London abgehaltenen Kongreß beide mit der Abfassung

wurden die Arbeiten an diesem Projekt eingestellt.

${ }^{3}$ Eine Ausnahme bildet Levenstein 1912. Er stellte Befragungen bei Arbeitern aus den wichtigsten Industriegebieten an und ließ sie Fragebögen ausfüllen, in denen sie darauf Antwort geben sollten, welche Bücher sie gelesen hätten. Eine Auswertung dieses Materials, in dem sich Hinweise auf die Kenntnis bestimmter Werke von Bebel, einzelner Werke von Marx und Engels ("Manifest der Kommunistischen Partei”, "Das Kapital”, „Der AntiDühring") sowie der Arbeiterpresse finden, hat unter sprachwissenschaftlichen Gesichtspunkten O. Basler ausgewertet. Vgl. Basler 1914.

4 Vgl. Autorenkollektiv 1978 sowie Autorenkollektiv 1981. 
eines für die Öffentlichkeit bestimmten Parteiprogramms und nannte sich seitdem „Bund der Kommunisten”. 1848 erschein das „Manifest der kommunistischen Partei”, die Geburtsurkunde des wissenschaftlichen Kommunismus.

In der 2. Hälfte des 19. Jh. wuchs in dem Maße, wie sich die industrielle Revolution mehr und mehr durchsetzte, die Arbeiterklasse an. Massen von Arbeitern konzentrierten sich in den Großstädten und industriellen Ballungsgebieten. Hier entstanden - jetzt auf deutschem Boden - neue Organisationen der Arbeiterbewegung, Arbeitervereine, deren Aufgabe die Interessenvertretung der Arbeiter gegenüber dem Arbeitgeber sein sollte. 1863 wurde in Leipzig der "Allgemeine Deutsche Arbeiterverein" (ADAV) gegründet, zu dessen Präsidenten F. Lassalle gewählt wurde. Politisches Hauptziel des Arbeitervereins, dessen Publikationsorgan der „Neue Sozialdemokrat" wurde, war es, das allgemeine, gleiche und direkte Wahlrecht zu erkämpfen. Auf dem 5. Vereinstag des Verbandes deutscher Arbeitervereine, der 1868 in Nürnberg tagte, setzten Bebel und Liebknecht die Annahme eines neuen Programms durch, das sich eng an die "Allgemeinen Statuten" der "Internationalen Arbeiterassoziation" (später I. Internationale) anschloß. Der Vereinstag faßte u.a. den Beschluß, verstärkt für die Bildung von Gewerkschaften zu wirken. Ende der sechziger Jahre war auch die Zeit herangereift für die Gründung einer Arbeiterpartei, die vor allem auch politische Ziele verfolgte. 1869 wurde in Eisenach die ${ }_{n}$ Sozialdemokratische Arbeiterpartei Deutschlands" gegründet, deren Programm von Bebel entworfen wurde. Es beruhte weitgehend auf dem des Nürnberger Vereinstages des Verbandes deutscher Arbeitervereine. Parteiorgan war der von Liebknecht in Leipzig herausgegebene „Volksstaat”.

Nach der Reichsgründung von 1871 wurde immer häufiger die Forderung erhoben, die Arbeiterorganisationen zu vereinigen, um noch schlagkräftiger die ökonomischen und politischen Interessen der Arbeiter vertreten zu können. 1875 gelang in Gotha die Vereinigung der „Sozialdemokratischen Arbeiterpartei Deutschlands" (Bebel) und des "Allgemeinen Deutschen Arbeitervereins" (Lassalle) zur "Sozialistischen Arbeiterpartei Deutschlands". Die Sozialdemokratie hatte nunmehr gesamtnationalen Charakter und gab dem Kampf der Arbeiterklasse Richtung und Ziel. Bebel und Liebknecht setzten sich als Führer mit einem Programm durch, das zwar wichtige sozialistische Prinzipien enthielt, aber hinter den Forderungen und Vorstellungen von Marx zurückblieb.

1878 trat das Sozialistengesetz, genauer gesagt das „Gesetz gegen die gemeingefährlichen Bestrebungen der Sozialdemokratie", in Kraft, durch das Bismarck nicht nur die Arbeiterklasse, sondern alle demokratischen 
und liberalen Kräfte politisch niederhalten wollte. Parteiorganisationen, Gewerkschaften, deren Zeitungen und Druckschriften wurden verboten. Genossenschaftsdruckereien wurden geschlossen, Versammlungen und andere öffentliche Veranstaltungen mit sozialdemokratischen Tendenzen wurden verboten. 1890 lehnte dann unter dem Druck einer stärker werdenden Arbeiterbewegung der deutsche Reichstag die Verlängerung des Sozialistengesetzes ab. Aus den Reichstagswahlen desselben Jahres gingen die Sozialdemokraten als stärkste Partei hervor.

Nach 1890 zog die deutsche Sozialdemokratie auf ihrem ersten Parteitag nach dem Fall des Sozialistengesetzes Bilanz ihres zwölfjährigen Kampfes, traf Vorbereitungen für ein neues Parteiprogramm und gab der Partei den neuen Namen "Sozialdemokratische Partei Deutschlands". 1891 wurde auf dem Erfurter Parteitag ein neues Parteiprogramm angenommen, das - durch revolutionäre Züge und sozialistische Grundsätze gekennzeichnet - den im "Manifest der kommunistischen Partei" durch Marx und Engels 1848 formulierten Vorstellungen relativ nahe steht.

Auch bei den Gewerkschaften kam es nach dem Fall des Sozialistengesetzes zu einer Neuorientierung. Die deutschen Gewerkschaften sollten reorganisiert werden, starke Zentralverbände sollten geschaffen werden, die eng miteinander zusammenarbeiteten. Zur Realisierung dieser Ziele wurde 1890 eine "Generalkommission der Gewerkschaften Deutschlands" als Koordinierungszentrum gebildet.

Soviel zum historischen Hintergrund. Die wenigen skizzenhaften Ausführungen sollen auch verdeutlichen, wie vielfältig die Richtungen und Strömungen in der Arbeiterbewegung waren, die bei einer sprachlichen Untersuchung des hier verwendeten politischen Wortschatzes berücksichtigt werden müßten.

\section{Zum Einfluß von Fremdsprachen auf den politischen Wort- schatz der Arbeiterbewegung}

Eines der wesentlichen Kennzeichen von Teilen des politischen Wortschatzes der Arbeiterbewegung besteht darin, daß Fremdsprachen wie das Englische und Französische sprachlich in unterschiedlicher Art und Weise auf seine Ausbildung eingewirkt haben.

Zum Einfluß des Französischen: In Frankreich waren bereits im 18. Jh. utopisch-kommunistische Ideen aufgekommen. In dieser Tradition stehend entwickelten zu Beginn des 19. Jh. Saint Simon (1760-1825) und Fourier (1772-1837) gesellschaftskritische Vorstellungen, Gesellschaftskonzeptionen, in denen Klassengegensätze durch Beispiel und Umerziehung überwunden werden sollten. Diese Ideen, vor allem die Lehre vom 
Klassenkampf, wurden in den dreißiger und vierziger Jahren von der sich in Deutschland formierenden Arbeiterbewegung aufgegriffen, vor allem natürlich von ihren Theoretikern.

Sprachlich wirkte sich das z.B. in Lehnbedeutungen aus, d.h. im Deutschen bereits vorhandene Wörter bildeten ein neues Semen nach französischem Vorbild aus. Ausbeutung, zunächst 'ertragreiche Ausschöpfung materieller und ideeler Werte' schloß an frz. exploitation an und wurde nun in der Arbeiterbewegung im Sinne von 'Aneignung fremder Arbeit durch den Besitzer der Produktionsmittel' gebräuchlich. Arbeiter, zunächst 'der mit der Hand Arbeitende' erhielt im Anschluß an frz. ouvrier - denkbar ist auch der Einfluß von engl. working man - die Bedeutung 'Angehöriger der sich entwickelnden Arbeiterklasse'. Das Antonym zu Arbeiter ist Kapitalist. Mitte des 17. Jh. in der Bedeutung 'reicher Bürger' von Kapital abgeleitet, wurde das Wort unter dem Einfluß von frz. capitaliste 'Angehöriger der Kapitalistenklasse' - so seit 1832 bei Blanqui - ebenfalls in dieser Bedeutung verwendet.

Proletarier, nach dem "Deutschen Wörterbuch" von J. und W. Grimm 'ein besitzloser, von der hand in den mund lebender mensch', ${ }^{5}$ im 18. Jh. nach lat. proletarius gebildet, erhielt nach 1830 unter dem Einflub von frz. prolétaire seine politische Bedeutung 'Angehöriger der sich entwickelnden Arbeiterklasse', wie sie bei Saint Simon vorgebildet war.

Frz. bourgeoisie 'Bürgertum, Bürgerstand' gelangte in der 2. Hälfte des 18. Jh. zunächst mit diesem hinsichtlich seiner sozialen Geltung nicht genau festgelegten Inhalt ins Deutsche. Im Anschluß an den Gebrauch, den die Saint-Simonisten im Französischen einführten, wurde das Wort nun bei den Theoretikern der Arbeiterbewegung, so auch bei Marx, im Sinne von 'kapitalistische Ausbeuterklasse' verwendet. Dasselbe gilt für frz. bourgeois ${ }_{n}$ Bürger", ursprünglich 'Bewohner einer Stadt' im Unterschied zum Adel und zur Landbevölkerung, das als Bourgeois ,'Bürger', seit der Mitte des 18. Jh. im Deutschen begegnet; seit der Mitte des 19. Jh. findet es sich dann - ebenfalls im Anschluß an saint-simonistischen Gebrauch - in Frankreich als 'Angehöriger der Kapitalistenklasse'.

Bereits Ende des 16. Jh. ist Classe, ${ }^{6}$ später Klasse, in der allgemeinen Bedeutung 'Abteilung, Gruppe mit gemeinsamen Merkmalen' belegt; es geht auf lat. classis zurück. Seit dem 18. Jh. stand Klasse auch für 'Menschengruppe innerhalb der Gesellschaft'. In England und Frankreich waren Historiker und Sozialökonomen zu der Erkenntnis gekommen, daß

${ }^{5}$ Grimm 1889, Sp. 2164.

6 Vgl. Autorenkollektiv 1989, H-P, S. 842 f. 
sich die Existenz von Klassen in der bürgerlichen Gesellschaft aus ökonomischen Verhältnissen erklärt; frz. classe bzw. engl. class wurden von ihnen dementsprechend definiert. Darauf aufbauend bestimmten Marx und Engels Klasse auch im Deutschen als 'große Gruppe von Menschen, die sich von einer anderen besonders nach ihrem Verhältnis zu den Produktionsmitteln und dadurch auch in der Erlangung und dem Umfang des Anteils am gesellschaftlichen Reichtum unterscheidet'.

Neben den Lehnbedeutungen existieren auch Lehnübersetzungen aus dem Vokabular der utopischen Sozialisten und französischer Historiker, d.h. Übersetzungen nach dem Muster französischer Wörter und Wendungen. Beipiele hierfür sind lutte des classes, das deutsch mit Klassenkampf wiedergegeben wurde, oder die Wendung dictature du prolétariat, die sich im Deutschen als Diktatur des Proletariats findet. Bei Arbeiterklasse ist nicht eindeutig zu entscheiden, ob es auf frz. classe des ouvriers oder engl. working class zurückgeht.

Vereinzelt wurden in der 1. Hälfte des 19. Jh. auch einzelne Wörter direkt aus dem Französischen übernommen, ohne daB eine Anpassung an das Deutsche erfolgte. Als Beispiel dafür kann Exploitation gelten, das bei den Saint-Simonisten die gängige Bezeichnung für 'Aneignung fremder Arbeit durch den Besitzer der Produktionsmittel' war. Marx z.B. bevorzugte in den 3 Bänden seines "Kapitals" Exploitation gegenüber synonymem Ausbeutung; es findet sich auch in einer relativ großen Zahl von Komposita als Determinativum, z.B. Exploitations: -feld, -form, grad, -mittel, -system, -theorie, -wut.

Zum englischen Einfluß: In England, wo sich die industrielle Revolution wesentlich früher als in Deutschland durchgesetzt hatte, erreichte die kapitalistische Entwicklung bereits zum Ende des 18. Jh. ein relativ hohes Niveau. In Übereinstimmung damit existierte auch eine hochentwickelte Lehre von den ökonomischen Verhältnissen unter kapitalistischen Bedingungen. A. Smith (1723-1790) und sein Schüler D. Ricardo (1772-1823) hatten wesentliche Gesetzmäßigkeiten erkannt, so z.B., daß die kapitalistische Wirtschaft nach objektiven ökonomischen Gesetzen funktioniert. Die klassische bürgerliche politische Ökonomie wurde in dem Maße, wie sich die industrielle Revolution im ersten Drittel des 19. Jh. auch in Deutschland zu entfalten begann, zu der entscheidenden Quelle, aus der die Theoretiker der sich formierenden Arbeiterbewegung schöpften, wenn sie die ökonomischen Verhältnisse auf deutschem Boden beschreiben und analysieren wollten.

So stellen einige zentrale Bezeichnungen der politischen Ökonomie in Deutschland Lehnübersetzungen dar, also Übersetzungen nach dem $\mathrm{Mu}$ - 
ster des Englischen. Wohl unter dem Einfluß von engl. those who live by wages, wie A. Smith die Klasse der den Reichtum der Gesellschaft vermehrenden Arbeiter nennt, entstehen im Deutschen die Komposita Lohnarbeitsleute (1776) und später Lohnarbeiter (1796), das in dt. ökonomischen Schriften seit den 20 er Jahren des 19 . Jh. geläufig wird. ${ }^{7}$ Nach dem Muster von engl. class of labourers und labouring class, working class wurde im Deutschen in der 2 . Hälfte des 18 . Jh. arbeitende Klasse und in den dreißiger Jahren des 19. Jh. Arbeiterklasse gebildet. Durch Marx, der die führende Rolle der Klasse der Lohnarbeiter im Kampf um die Befreiung von der Ausbeutung erkannte, fand Arbeiterklasse schnell Verbreitung. Mit direktem Bezug auf englische Verhältnisse wurde in den vierziger Jahren des 19. Jh. Arbeiterbewegung gebildet. Vorbild war engl. working-men's movement, das sich seinerseits an frz. parti du mouvement anlehnte.

Auch Lehnbedeutungen entstanden unter englischem Einfluß. Bei Gewerkschaft, dem Fachwortschatz der Bergleute entstammend und seit dem 16. Jh. in der Bedeutung 'Gesamtheit aller Teilhaber an einem Bergwerk' belegt, bildete sich nach dem Vorbild von engl. trade union die seit 1868 bezeugbare Bedeutung 'organisatorischer ZusammenschluB der Arbeiter zur Vertretung und Durchsetzung ihrer ökonomischen und politischen Ziele' aus. Demonstration, seit dem 16. Jh. in der Bedeutung 'Darlegung' nachweisbar, entwickelte unter dem EinfluB von engl. demonstration die Lehnbedeutung 'politische Willensbekundung, öffentliche Kundgebung zur Durchsetzung politischer Ziele'.

Ein markantes Beispiel für die direkte Übernahme eines Wortes aus dem Englischen, das dem politischen Wortschatz der Arbeiterbewegung angehört, stellt Streik dar in der Bedeutung 'Arbeitsniederlegung mit dem Ziel der Durchsetzung ökonomischer und politischer Ziele, Ausstand'. Es geht auf engl. to strike zurück, das seit 1810 in dieser Verwendung bezeugt ist. Die Bezeichnung, die bis 1890 noch in der englischen Schreibweise Strike begegnet, bezog sich zunächst auf englische Verhältnisse, wurde aber seit dem Leipziger Buchdruckerstreik 1865 auch auf Arbeitskämpfe in Deutschland angewendet und fand danach schnell Verbreitung. Wie einer distanzierenden Bemerkung Bismarcks von $1878 \mathrm{zu}$ entnehmen ist, galt das Wort damals noch als Ausdruck der Sprache der Arbeiter, ging dann aber schnell in den Wortschatz der Allgemeinsprache ein.

7 Vgl. Autorenkollektiv 1989, A-G, S. 71. 


\section{Zur Herausbildung von Fachwortschätzen im politischen Wortschatz der Arbeiterbewegung}

Zu den charakteristischen Erscheinungen in der Entwicklung des politischen Wortschatzes der Arbeiterbewegung gehört die Herausbildung von Fachwörtern (Termini) und damit von Fachwortschätzen, die sich in bestimmten wissenschaftlichen Sachbereichen der Philosophie, Ökonomie und der Gesellschaftslehre vollzog. Mit Fachwörtern können wissenschaftliche Einheiten geordnet und klassifiziert werden, mit ihrer Hilfe lassen sich wissenschaftliche Erkenntnisse prägnant zusammenfassen. Sie sind in der Regel definiert und besitzen innerhalb eines wissenschaftlichen Systems einen bestimmten Stellenwert.

Im Prozeß der Ausarbeitung der Theorie standen für ein Denotat in der Regel mehrere mehr oder weniger synonyme Bezeichnungen zur Verfügung, von denen in den jeweiligen Theorien bestimmte ausgewählt und zum Träger des terminologischen Gehalts, also definiert wurden. Bevor auf den Terminologisierungsprozeß selbst anhand einzelner Beispiele eingegangen wird, soll zunächst an einigen Denotaten gezeigt werden, aus welcher sprachlichen Vielfalt dabei ausgewählt werden konnte:

/Arbeiter/: $\quad$ Arbeiter, Arbeitsmann, Arbeitnehmer, Arbeitssklave, Fabriksklave, Lohnarbeiter, Lohnsklave, Prolet, Proletar, Proletarier

/Arbeiterklasse/: Arbeiterklasse, Arbeiterschaft, Arbeiterstand, arbeitende Hände, arbeitende Klasse, Proletariat

/Ausbeutung/: Ausbeutung, Ausnutzung, Auspowerung, Auspumpung, Aussaugung, Exploitation

/proletarisch/: kommunistisch, proletarisch, revolutionär, rot, sozialistisch

/Revolution/: Aufstand, Revolution, Umwälzung, Umsturz

Innerhalb des Denotatsbereichs/Arbeiterklasse/ war z.B. in den Lehren der utopischen Sozialisten, speziell bei Lassalle, Arbeiterstand der zentrale Terminus. Das wird auch darin deutlich, daß er ihn eindeutig gegenüber Proletariat bevorzugte. Vgl. folgende Definition aus einer seiner Reden:

es [wird] ... nöthig sein, uns klar zu werden über das, was wir denn eigentlich unter „Arbeiter” oder „Arbeiterstand” verstehen. ... Arbeiter sind wir alle, insofern wir nur eben den Willen haben, uns in irgend einer Weise der menschlichen Gesellschaft nützlich zu machen. Dieser 
vierte Stand ... ist eben deshalb gleichbedeutend mit dem ganzen Menschengeschlecht. ${ }^{8}$

Proletariat - Bourgeoisie bei Marx entsprach daher bei Lassalle Arbeiterstand - Bürgertum. ${ }^{9}$

Sieht man sich die Werke von Marx und Engels an, so fält zunächst die Vielfalt der Bezeichnungen auf, die für den Denotatsbereich/Arbeiterklasse/ verwendet wurden. 1845 hob Engels in seiner Schrift "Die Lage der arbeitenden Klasse in England" sogar hervor, daß er die Ausdrücke Arbeiter (working men) und Proletarier, Arbeiterklasse, besitzlose Klasse und Proletariat fortwährend als gleichbedeutend gebraucht. ${ }^{10}$ Im Zuge der weiteren Arbeit an ihrem terminologischen System begann dann ein Prozeß, in dessen Verlauf einige Synonyme aufgegeben wurden. Seit dem "Manifest der kommunistischen Partei” von 1848 waren dann Proletariat und Arbeiterklasse Kernwörter dieses Denotatsbereichs. Vgl. dazu folgenden Ausschnitt aus dieser programmatischen Schrift:

Geschlechts- und Altersunterschiede haben keine gesellschaftliche Geltung mehr für die Arbeiterklasse. Es gibt nur noch Arbeitsinstrumente, die je nach Alter und Geschlecht verschiedene Kosten machen. ... Von allen Klassen, welche heutzutage der Bourgeoisie gegenüberstehen, ist nur das Proletariat eine wirklich revolutionäre Klasse. ${ }^{11}$

Während Proletariat überwiegend in den politisch-agitatorischen, programmatischen und historischen Schriften verwendet wurde, machten sie im ökonomischen Schrifttum vor allem von Arbeiterklasse Gebrauch. Thr Einsatz war also von der Textsortenspezifik abhängig. Nach 1860 trat dann Proletariat gegenüber Arbeiterklasse allmählich in den Hintergrund. Insgesamt wurde Proletariat von Engels häufiger gebraucht als von Marx, aber ab etwa 1880 benutzte auch Engels fast ausschließlich Arbeiterklasse.

Betrachtet man die Programme der drei im letzten Drittel des 19. Jh. gegründeten Arbeiterparteien, so ergibt sich folgendes Bild. Im Programm der 1869 in Eisenach gegründeten "Sozialdemokratischen Arbeiterpartei Deutschlands", die noch stark unter dem Einfluß der Lassalleaner stand, verwendete man zur Bezeichnung der Arbeiterklasse arbei-

${ }^{8}$ Lassalle 1891, S. 176.

${ }^{9}$ Vgl. Conze 1972, S. $216 f$.

10 Marx/Engels Bd. 2, S. 234.

11 Marx/Engels Bd. 4, S. 469-472. 
tende Klasse und Arbeiterklasse selbst. Man vgl.:

Der Kampf für die Befreiung der arbeitenden Klassen ist nicht ein Kampf für Klassenprivilegien und Vorrechte, sondern für gleiche Rechte und gleiche Pflichten und für die Abschaffung aller Klassenherrschaft ${ }^{12}$

sowie

In Erwägung, daß die politische und ökonomische Befreiung der Arbeiterklasse nur möglich ist, wenn diese gemeinsam und einheitlich den Kampf führt, gibt sich die Sozialdemokratische Arbeiterpartei eine einheitliche Organisation. ${ }^{13}$

Auch im Parteiprogramm der "Sozialistischen Arbeiterpartei Deutschlands", in der sich 1875 in Gotha "Lassalleaner" und "Eisenacher" vereinigten, war der Einfluß des lassalleanischen Ideengutes noch relativ stark. Im Gothaer Programm wurde nur Arbeiterklasse gebraucht. Vgl. dazu:

In der heutigen Gesellschaft sind die Arbeitsmittel Monopol der Kapitalistenklasse; die hiendurch bedingte Abhängigkeit der Arbeiterklasse ist die Ursache des Elends und der Knechtschaft in allen Formen. ${ }^{14}$

Nach Aufhebung des Sozialistengesetzes 1890 zog die deutsche Sozialdemokratie Bilanz. Das Ergebnis war 1891 ein neues Programm, das den Vorstellungen von Marx und Engels stärker verpflichtet war, und ein neuer Name: „Sozialdemokratische Partei Deutschlands". Arbeiterklasse dominiert in diesem Programm. Vgl.: Die Befreiung der Arbeiterklasse ist also ein Werk, an dem die Arbeiter aller Kulturländer gleichmäßig beteiligt sind. ${ }^{15}$ Aber vereinzelt begegnet auch Proletariat bzw. Proletarier. Vgl.:

Diese gesellschaftliche Umwandlung bedeutet die Befreiung nicht blo $\beta$ des Proletariats, sondern des gesamten Menschengeschlechts, das unter den heutigen Zuständen leidet. Aber sie kann nur das Werk der Arbeiterklasse sein. ${ }^{16}$

Das bestätigt die Feststellung von W. Conze in seinem Artikel "Proletariat, Pöbel, Pauperismus" in "Geschichtliche Grundbegriffe. Hi-

${ }_{12}$ Berthold/Diehl 1967, S. 45.

${ }^{13}$ Berthold/Diehl 1967, S. 45.

14 Berthold/Diehl 1967, S. 47.

15 Berthold/Diehl 1967, S. 84.

${ }^{16}$ Berthold/Diehl 1967, S. 83. 
storisches Lexikon zur politisch-sozialen Sprache in Deutschland", ${ }^{17}$ da 8 Proletariat/Proletarier im letzten Drittel des 19. Jh. - offenbar unter dem Einfluß des "Manifests der kommunistischen Partei" - zunehmend bevorzugt wurde. Das gilt auch für die Anhänger Lassalles, die 1869/70 in Augsburg eine Arbeiterzeitung unter dem Titel „Der Proletarier” herausgaben.

\section{Zur Entwicklung von Fachwörtern des politischen Wortschat- zes der Arbeiterbewegung}

Innerhalb des politischen Wortschatzes der Arbeiterbewegung kam es bei den definierten Fachwörtern, z.B. den den Kerngehalt der Lehre vom Klassenkampf tragenden Termini in dem Maße zu Veränderungen, wie Marx und Engels, ausgehend von utopisch-sozialistischen Vorstellungen, diese Lehre weiterentwickelten. Das soll am Beispiel von Proletariat nachgezeichnet werden.

Proletariat, das Mitte der dreifiger Jahre des 19. Jh. schlagartig im Zusammenhang mit der Diskussion um den Pauperismus auftrat, wies als Terminus des utopischen Sozialismus mit der Bedeutung 'die sich entwickelnde Klasse der Armen, Besitzlosen, der Proletarier' folgende Merkmalsstruktur auf:

(1) die Klasse der Armen, Elenden, Besitzlosen

(2) die Klasse der Abhängigen, Ausgebeuteten

(3) die sich vereinigende, ein gemeinsames Bewußtsein entwickelnde Klasse

(4) die Klasse der Aufrührerischen, Revolutionären.

Das Merkmal 'Klasse der Armen, Elenden, Besitzlosen' dominiert.

Marx und Engels entwickelten in den vierziger Jahren die Lehre vom Klassenkampf weiter. Im "Manifest der kommunistischen Partei" von 1848 legten sie die erste zusammenfassende Darstellung des wissenschaftlichen Kommunismus vor. Danach verläuft die historische Entwicklung gesetzmäßig und führt zum Sturz des Kapitalismus und zum Sieg des Sozialismus/Kommunismus in der ganzen Welt. Die gesellschaftliche Triebkraft dieser gesetzmäßigen Entwicklung ist der Klassenkampf. Der Arbeiterklasse, dem Proletariat, fallt die welthistorische Aufgabe zu, eine neue, klassenlose, von Ausbeutung und Unterdrückung freie Gesellschaft

${ }^{17}$ Vgl. Conze 1984, S. 27ff. 
zu schaffen. Da die Stellung des Proletariats in der kapitalistischen Gesellschaft und die sich daraus ergebenden Klasseninteressen und Ziele überall gleich sind, lautete die programmatische Losung des Manifests: „Proletarier aller Länder, vereinigt euch”. In diesem Kontext wurde nun Proletarier neu definiert und wies als Terminus mit der Bedeutung 'die Klasse der Unterdrückten im Kapitalismus' folgende Merkmalstrukturen auf:

(1) die Klasse der Besitzlosen an Produktionsmitteln

(2) die Klasse der doppelt freien Lohnarbeiter, der Ausgebeuteten

(3) die Vereinigten, Klassenbewußten

(4) die Errichter der Diktatur des Proletariats.

Jetzt dominiert nicht mehr das Merkmal 'die Klasse der Armen, Elenden, Besitzlosen', sondern das Merkmal 'die Errichter der Diktatur des Proletariats'. Diese Bedeutung findet sich in folgenden Ausschnitten aus dem Manifest:

In demselben Maße, worin sich die Bourgeoisie, d.h. das Kapital, entwickelt, in demselben Maße entwickelt sich das Proletariat, die Klasse der modernen Arbeiter, die nur so lange leben, als sie Arbeit finden, und die nur so lange Arbeit finden, als ihre Arbeit das Kapital vermehrt. Diese Arbeiter, die sich stückweise verkaufen müssen, sind eine Ware wie jeder andere Handelsartikel. ${ }^{18}$

Von allen Klassen, welche heutzutage der Bourgeoisie gegenüberstehen, ist nur das Proletariat eine wirklich revolutionäre Klasse. Die übrigen Klassen verkommen und gehen unter mit der großen Industrie, das Proletariat ist ihr eigenstes Produkt. ${ }^{19}$

Der nächste Zweck der Kommunisten ist derselbe wie der aller übrigen proletarischen Parteien: Bildung des Proletariats zur Klasse, Sturz der Bourgeoisieherrschaft, Eroberung der politischen Macht durch das Proletariat. ${ }^{20}$

5. Zum Eindringen von Elementen des politischen Wortschatzes der Arbeiterbewegung in den Wortschatz der Allgemeinsprache

Generell ist davon auszugehen, daß der politische Wortschatz der Arbeiterbewegung zunächst wohl nur bei den aus bürgerlichen Kreisen stam-

$18 \mathrm{Marx} /$ Engels Bd. 4, S. 468.

19 Marx/Engels Bd. 4, S. 472.

20 Marx/Engels Bd. 4, S. 474. 
menden Theoretikern der Arbeiterbewegung, in Kreisen der Arbeiter sowie mit ihnen Sympathisierender gebräuchlich war. Das gilt besonders für die politischen Termini, deren theoretischer Gehalt wohl weitgehend nur den führenden Köpfen bekannt war. Ihr Gebrauch, die umfassende Realisierung ihres theoretischen Gehalts, setzte einen wissenschaftlichen Horizont voraus, der dem Arbeiter im Normalfall aufgrund der Verhältnisse im Volksschulwesen fehlen muBte.

Schrittweise fand dieser politische Wortschatz jedoch auch Eingang in den Wortschatz der Allgemeinsprache. Für seine Verbreitung in der Arbeiterbewegung - die Termini eingeschlossen - sorgte zum einen die Arbeiterpresse. Erwähnt seien das von Liebknecht herausgegebene "Demokratische Wochenblatt" (1868/69), der „Volksstaat" (1869/76), der „Vorwärts" (1876/78), aber auch sozialdemokratische Regionalzeitungen wie ${ }_{n}$ Das Schlesische Tageblatt" oder die "Sächsische Arbeiterzeitung", in der Zeit des Sozialistengesetzes der "Sozialdemokrat", der $1879 / 88$ zunächst in Zürich, anschließend bis 1890 in London erschien, illegal nach Deutschland eingeführt und verbreitet wurde. Zum anderen trugen auch Schriften agitatorisch-propagandistischen Charakters führender Persönlichkeiten der Arbeiterbewegung zu seiner Verbreitung bei. Das Interesse an politischer Information wuchs gerade in der Zeit des Verbots der Sozialdemokratie stark an. Während die Sozialdemokraten sowie die Gewerkschaften 1878 über 56 Presseerzeugnisse mit rund 160.000 Lesern verfügten, waren es 1890, nach der Aufhebung des Sozialistengesetzes, über 100 Presseorgane mit ca. 600.000 Lesern. Diese Steigerung ist umso bemerkenswerter, als während dieser Zeit so gut wie alle Druckerzeugnisse im Ausland hergestellt und illegal eingeführt werden mußten, also trotz damit verbundener Gefahren ein illegaler Zeitungsund Literaturvertrieb auf- und ausgebaut wurde.

Zu den Schriften führender Vertreter der Arbeiterbewegung, die auf besonderes Interesse stießen, gehörte z.B. Bebels 1883 erschienenes Werk "Die Frau und der Sozialismus", das Rekordauflagen erlebte. 1902 erschien die 33., 1910 die 50. Auflage. Zwei Beispiele, das eine aus einer Zeitung, das andere aus einer Schrift Rosa Luxemburgs, sollen - stellvertretend für andere - die Verbreitung sozialdemokratischen Gedankengutes belegen:

Demokratisches Wochenblatt (1869)

Das Kapital ist international, darum muß der Kampf gegen das Kapital international geführt werden - Proletarier aller Länder vereinigt euch. $^{21}$

21 Demokratisches Wochenblatt 1868, S. 270a. 
Nach der Marxschen Lehre führt die ökonomische Entwicklung in der modernen Gesellschaft zum Untergang des selbstwirtschaftenden Arbeiters und zu seiner Verwandlung in einen Lohnarbeiter, der von dem Kapitalisten ausgebeutet wird. ... [Die] Konzentration der Kapitalien bildet nach Marx ... die materielle Grundlage ... zur Aufhebung der kapitalistischen Gesellschaft. ... Sie stellt die historische Aufgabe: Einführung einer sozialistischen Gesellschaftsordnung: sie produziert die Kräfte zur Lösung der Aufgabe: die Proletarier. ${ }^{22}$

Darüber hinaus ist jedoch davon auszugehen, daß Teile des politischen Wortschatzes der Arbeiterbewegung allmählich auch bei Nichtarbeitern bekannt wurden. Zumindest ist eine passive Kenntnis wesentlicher Bezeichnungen anzunehmen. J. Grimm z.B. führte in der 3. Lieferung des "Deutschen Wörterbuchs" beim Artikel „Arbeitskraft" an: ${ }^{23}$

man betrachtet den menschen mit seiner arbeitskraft wie eine waare, deren preis mit der menge des angebots und der nachfrage danach steigt und fällt.

Auch bekannte Konversationslexika wie der „Brockhaus” oder "Meyers Konversationslexikon" buchten politischen Wortschatz der Arbeiterbewegung, speziell auch Termini wie Lohnarbeit, Kapital, Arbeiterklasse, arbeitende Klasse.

Brockhaus (14. Auflage 1894)

ein Gegensatz zwischen kapitalbesitzenden Unternehmern und kapitallosen Lohnarbeitern war [im Mittelalter] noch nicht vorhanden. ... Die moderne Fabrikindustrie ... schuf aber nicht nur eine neue ... Arbeiterklasse, sondern ... stellte Arbeitgeber und $A$ [rbeiter] als Fremde einander gegennüber, die Kluft zwischen Arbeit und Kapital erweitert sich mehr und mehr. ... Somit versteht man gegenwärtig unter $A$ [rbeiter] etwas ganz anderes als vor hundert ... Jahren. Erst die moderne Großindustrie hat den heutigen Arbeiterstand geschaffen, und erst auf dem Boden der kapitalistischen Gütererzeugung sind die mannigfachen Fragen erwachsen, welche sich auf die Verbesserung der Lage der arbeitenden Klassen beziehen. ${ }^{24}$

Meyers Konversationslexikon (6. Auflage 1904)

... ist es üblich geworden, den Begriff Arbeiter etwas enger zu fassen, indem man (so insbes. die Sozialisten) darüber die Klasse der Lohnarbeiter (arbeitende Klassen, Arbeiterstand) im Gegensatz zu den ...

22 Luxemburg 1970, S. 538.

23 Grimm 1854, Sp. 544.

24 Brockhaus 1894, Sp. 812a. 
Kapitalisten versteht. ${ }^{25}$

Außerdem trugen auch bürgerliche Presseorgane, die sich von ihrem Standpunkt mit der Arbeiterfrage auseinandersetzten, dazu bei, daß sich der politische Wortschatz der Arbeiterbewegung auch in der Allgemeinsprache verbreitete. Die ${ }_{n}$ Neue Preußische Zeitung" z.B., auch "Kreuzzeitung" genannt, veröffentlichte 1891 eine Artikelreihe „Diktatur des Proletariats", in der sie sich mit der Sache und damit mit der entsprechenden Wendung auseinandersetzte. Dabei ließ sie natürlich - entsprechend ihrer politischen Position - keine Gelegenheit ungenutzt, sich vom Inhalt, wie er in großen Teilen der Arbeiterbewegung galt, zu distanzieren, und bemühte sich, negative Emotionen auszulösen. Vgl. dazu folgenden Ausschnitt:

Was die „Übergangsperiode einer revolutionären Diktatur des Proletariats" bedeutet, darüber hat die Pariser Kommune von 1871 Aufschluß gegeben. ${ }^{26}$

Mit dem Eindringen von Wörtern des politischen Wortschatzes der Arbeiterbewegung, also auch der Termini, in den Wortschatz der Allgemeinsprache, d.h. mit der zunehmenden Häufigkeit ihres Gebrauchs in allen Klassen und Schichten des deutschen Volkes war verbunden, daB sie auch metaphorisch eingesetzt werden konnten. Für die Termini bedeutete das einen Prozeß der Entterminologisierung. W. Fleischer hat diesen Vorgang folgendermaßen beschrieben: Wenn die Stufe einer gewissen Aligemeinverständlichkeit, Geläufigkeit und Vertrautheit erreicht ist, „so kann - vom passiven Verständnis zum aktiven Gebrauch - eine neue Verwendung und damit Entterminologisierung eintreten", dann "können Termini in Situationen des alltäglichen Lebens übertragen und mit Bedeutungen verwandt werden, die nicht mehr der terminologischen Bedeutung entsprechen."27

In dem Maße, wie sich bürgerliche Kreise mit dem politischen Anliegen der Arbeiterbewegung auseinandersetzten, kam es auch dazu, daß jetzt eine Bezeichnung - je nach politischem Standpunkt des Sprechers/Schreibers - unterschiedlichen Denotatsbezug aufweisen konnte (Homonymie), daß sich neue Sememe eines Lexems ausbildeten (Polysemie) oder daß Denotate unterschiedlich bewertet wurden. Während bei Proletarier - wie schon gezeigt - im Sprachgebrauch revolutionärer Teile der Arbeiterklasse das Merkmal 'die Errichter der Diktatur des Proletari-

25 Meyers 1904, Sp. 673b.

26 Neue Preußische Zeitung 1891, S. 3a.

27

Vgl. Fleischer 1969, S. 481. 
ats' dominant war, konnte in anderen Fällen, z.B. bei Nichtarbeitern, das Merkmal 'Klasse der Armen, Elenden, Besitzlosen' vorherrschen. Vgl. dazu folgenden Ausschnitt aus "Der Demokrat" von 1850:

... daß die sicher vorschreitende Entwerthung der Arbeitskraft eine im. mer weitere Ausdehnung der traurigen Masse des Proletariats, einer abhängigen, entwürdigten, um die bloße Existenz kämpfenden Klasse ist, das kann nicht oft genug gesagt werden. ${ }^{28}$

Insgesamt war die Folge, daß es seit dem letzten Drittel des 19. Jh. im ideologierelevanten Wortschatz der Allgemeinsprache verstärkt zu semantischen Differenzierungen kam, die von den jeweiligen politischen Positionen des Sprechers/Schreibers abhängig waren.

\section{Literatur}

Autorenkollektiv (1978): Zum Einfluß von Marx und Engels auf die deutsche Literatursprache. Studien zum Wortschatz der Arbeiterklasse im 19. Jahrhundert. Von einem Autorenkollektiv unter der Leitung von Joachim Schildt. Berlin (Bausteine zur Sprachgeschichte des Neuhochdeutschen, Bd. 59).

Autorenkollektiv (1981): Die Auswirkungen der industriellen Revolution auf die deutsche Sprachentwicklung im 19. Jahrhundert. Von einem Autorenkollektiv unter der Leitung von Joachim Schildt. Berlin (Bausteine zur Sprachgeschichte des Neuhochdeutschen, Bd. 60).

Autorenkollektiv (1989): Etymologisches Wörterbuch des Deutschen. Von einem Autorenkollektiv unter der Leitung von Wolfgang Pfeiffer. Berlin.

Basler, O. (1914): Die Sprache des modernen Arbeiters. Ein Versuch ihrer Darstellung. In: Zeitschrift für deutsche Wortforschung. Hrsg. von F. Kluge. Bd. 15. Strabburg, S. 246-270.

Berthold, L./Diehl, E. (Hrsg.) (1967): Revolutionäre deutsche Parteiprogramme. Vom Kommunistischen Manifest zum Programm des Sozialismus. Berlin.

Brockhaus (1894): Brockhaus' Konversations-Lexikon. 14. Aufl. Bd. 1. Leipzig/Berlin/Wien.

Brunner, O./Conze, W./Koselleck, R. (Hrsg.) (1972-1984): Geschichtliche Grundbegriffe. Historisches Lexikon zur politisch-sozialen Sprache in Deutschland. 5 Bde. Stuttgart.

Conze, W. (1972): Arbeiter. In: Brunner/Conze/Koselleck. Bd. 1.

28 Der Demokrat 1850, S. $97 \mathrm{~b}$. 
Conze, W. (1984): Proletariat, Pöbel, Pauperismus. In: Brunner/Conze/ Koselleck. Bd. 5.

Demokratisches Wochenblatt (1868): Demokratisches Wochenblatt. Organ der Deutschen Volkspartei und des Verbandes Deutscher Arbeitervereine. Leipzig 1868 und 1869. Fotomechanischer Nachdruck Leipzig 1969, 1971.

Der Demokrat (1850): Der Demokrat. 2. Jg. Weimar.

Fleischer, W. (1969): Terminologie und Fachsprache im Bereich der Politik. In: Wissenschaftliche Zeitschrift der Pädagogischen Hochschule Potsdam. Gesellschafts- und sprachwissenschaftliche Reihe. 13. Jg. Potsdam.

Grimm, Jacob/Grimm, Wilhelm (1854): Deutsches Wörtbuch. Bd. 1. Leipzig.

Grimm, Jacob/Grimm, Wilhelm (1889): Deutsches Wörterbuch. Bd. 13. Leipzig.

Lassalle, Ferdinand (1891): Ausgewählte Reden und Schriften. Bd. 1. Leipzig.

Levenstein, A. (1912): Die Arbeiterfrage. München.

Luxemburg, Rosa (1970): Gesammelte Werke. Bd. 1, 1. Halbband. Berlin.

Marx, Karl/Engels, Friedrich: Werke. Bd. 2. Berlin.

Meyers (1904): Meyers Großes Konversations-Lexikon. 6. Auf. Bd. 1. Leipzig/Wien.

Neue Preußische Zeitung (1891): Neue Preußische Zeitung. Jg. 1891, Nr. 61. Berlin. 